\title{
EFFECT OF OPTICAL FILTERING ON 20-GBIT/S RZ-DQPSK TRANSMISSION OVER 2000 KM IN A 64-CHANNEL DWDM SYSTEM
}

\author{
Pierpaolo Boffi ${ }^{1}$, Lucia Marazzi ${ }^{1}$, Paolo Martelli ${ }^{1}$, Livio Paradiso ${ }^{1}$, Paola Parolari ${ }^{1}$, \\ Aldo Righetti ${ }^{1}$, Rocco Siano ${ }^{1}$, and Mario Martinelli ${ }^{1.2}$ \\ ${ }^{\prime}$ CoreCom, via G. Colombo, 81, 20133 Milano, Italy boffi@corecom.it \\ ${ }^{2}$ Politecnico di Milano, piazza L. da Vinci 32, 20133 Milano,
}

\begin{abstract}
We analyze 20-Gb/s RZ-DQPSK transmission in a $33-\mathrm{GHz}$ spaced 64-channel DWDM system over a $2000-\mathrm{km}$ laboratory straight line. In particular filtering impact on DQPSK modulation format due to optical filter and a de-interleaver at the receiver and pre-filter at the transmitter are experimentally evaluated and commented.
\end{abstract}

\section{INTRODUCTION}

Nowadays there is a growing effort to upgrade the transmission capacity of optical systems by increasing the data transmission spectral efficiency. For this purpose modulation formats alternative to usual IMDD are explored [1,2]. Optical differential quadrature phase shift keying (DQPSK) format appears very promising, as it combines increased information rate due to multi-level modulation with the simplicity of direct detection. Recently a few papers have presented experimental applications of this format, showing also DQPSK robustness toward fiber nonlinear effects $[3,4,5]$ and DQPSK employment in submarine systems designed for standard IMDD transmission [6].

It is also known that optical communication channel performance is strongly influenced by the receiver optical filter characteristics as well as by the choice of the optical pre-filter. Of course this effect varies with the signal modulation format $[7,8]$. 
In this contribution we experimentally analyze the impact of the filtering apparatus on the BER performance in a transmission of a RZ-DQPSK signal at 10 Gsymbol/s (20 Gb/s equivalent) in a 64-channel DWDM system with $33-\mathrm{GHz}$ spacing over $2000 \mathrm{~km}$. In particular at the receiving unit we analyze the combined effect of a $33 / 66 \mathrm{GHz}$ de-interleaver followed by an optical tunable band pass filter, while at the transmission side we study the effect on DWDM long-haul propagation of pre-filtering. The preliminary experimental results obtained with non-optimized filters clearly show the importance of an accurate project for filter shapes and bandwidths.

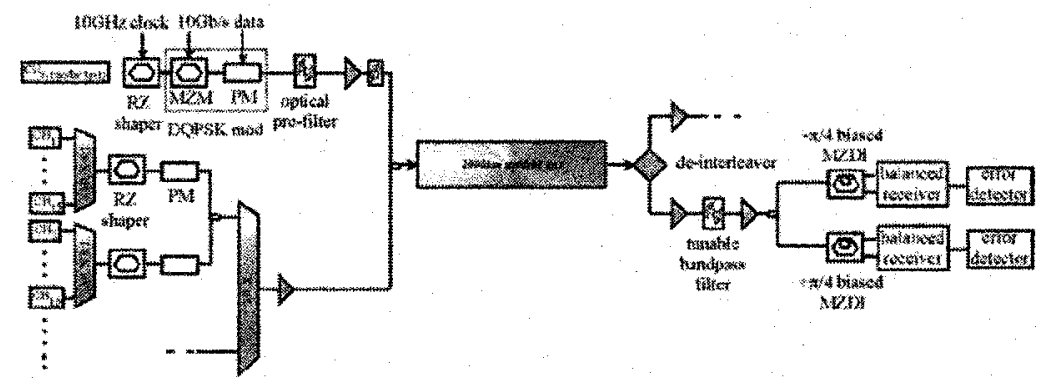

Figure 1. Experimental setup.

\section{EXPERIMENTAL SET UP}

The experimental setup is presented in Figure 1. The 64 channels, 33-GHz spaced from $1543.03 \mathrm{~nm}$ to $1559.71 \mathrm{~nm}$, are multiplexed in a $33-\mathrm{GHz}$ grid. A tunable laser generates the DQPSK channel under test. RZ-DQPSK modulation is obtained by the cascade of a first RZ-shaper, a Mach-Zehnder modulator (MZM) and a phase modulator (PM). The RZ-shaping is performed by a Mach-Zehnder modulator (MZM) driven by the $10 \mathrm{GHz}$ clock signal, obtaining a duty-cycle of $50 \%$ and an extinction ratio of $13 \mathrm{~dB}$. The second MZM operates in push-pull mode and is driven by a $10-\mathrm{Gb} / \mathrm{s}$ PRBS of length $2^{7}-1$ achieving a $\pi$-modulation depth. The last PM, driven by the complementary PRBS signal suitably delayed in order to obtain uncorrelation, performs an additional $\pi / 2$-phase modulation. An RF phase shifter synchronizes data at the two phase modulators. The test channel is inserted after the multiplexer.

Setup allows RZ shaping of the other 63 channels, which are $0-\pi$ binary phase shift keyed (BPSK) by a single PM. Due to the same RZ format and to the BPSK modulation with $\pi$-depth, the obtained 63 channels optical spectra are very similar to the RZ-DQPSK channel under test, as demonstrated in [6]. The RZ-DQPSK 
channel under test can be pre-filtered before propagation by means of a $22 \mathrm{GHz}$ filter. Actually employed pre-filter is a $66 / 33$ interleaver, whose optical transfer function is shown in Figure 2. Receiver de-interleaver has the same transfer function and demultiplexes to $66 \mathrm{GHz}$ the $33 \mathrm{GHz}$ DWDM grid. A tunable band pass filter (FWHM of $33 \mathrm{GHz}$ ) selects the DQPSK channel under test for BER measurements. The in-phase and in-quadrature phases are directly detected by a pair of Mach-Zehnder delay interferometers (MZDI) biased at $+\pi / 4$ and $-\pi / 4$, respectively [9].

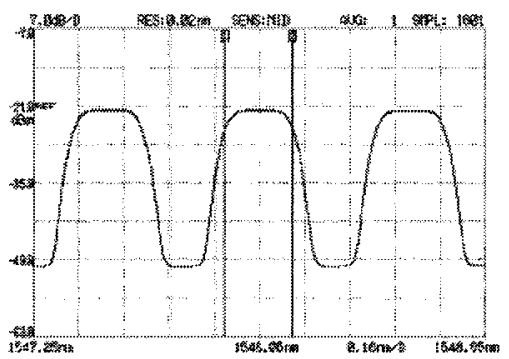

Figure 2. Measured optical transfer function of the employed optical pre-filter, which is an interleaver with the same spectral characteristics of the receiver interleaver.

We employ a pair of originally designed MZDIs [10] realized by SiON technology with a high index contrast between the guide core and upper cladding/substrate, allowing minimum bending radius with negligible losses in the $100 \mathrm{ps}$ delay arm. In order to set the differential optical phase between the interferometer arms respectively to $+\pi / 4$ and $-\pi / 4$, the integrated SiON chips are thermally controlled by a heater inside a metallic case. The two outputs of each MZDI are detected by a commercially available balanced receiver $(14-\mathrm{GHz}$ bandwidth), mechanically controlling the optical path lengths of the balanced receiver. Then the BER-tester is programmed with the expected data sequence of $2^{7}-1$ bits.

The DWDM channels copropagate over a 1994-km straight line constituted by 33 NZD fiber spans of $55 \mathrm{~km}$ compensated, every 6 spans, by SMR fibers. NZD fiber has a negative dispersion of $-2.82 \mathrm{ps} / \mathrm{nm} \mathrm{km}$ at $1548 \mathrm{~nm}$, the link average dispersion at $1548 \mathrm{~nm}$ is $0.0488 \mathrm{ps} / \mathrm{nm} \mathrm{km}$. In the straight line 39 EDFAs with $17-$ $\mathrm{nm}$ flat optical bandwidth, $12-\mathrm{dBm}$ saturation output power and 4.2-dB noise figure are employed. This laboratory link was originally designed for an IMDD submarine transmission by Pirelli Submarine Telecom Systems. 


\section{EXPERIMENTAL RESULTS AND DISCUSSION}

DQPSK performance are evaluated in the above-described $2000 \mathrm{~km}$ DWDM system in order to understand design complexity and limitations due to filtering specifications over propagation impairments. In the following figures we show BER measurements relative to $+\pi / 4$ biased MZDI receiver; similar results are obtained for $-\pi / 4$ biased MZDI.

In particular we investigate BER performances of the RZ-DQPSK channel at $10 \mathrm{Gsymbol} / \mathrm{s}$ as a function of system optical signal-to-noise ratio (OSNR), at $\lambda=1548 \mathrm{~nm}$. First of all, Figure 3 shows curves comparison between unfiltered (black diamonds) and narrow filtered (gray squares) RZ-DQPSK. It can be noticed that RZ-DQPSK signal appears robust towards narrow-band filtering induced by the de-interleaver at the receiver. Moreover, the penalty due to the detuning of the signal wavelength with respect to the de-interleaver optical transfer function remains less than $1 \mathrm{~dB}$ for detuning within $+/-7.5 \mathrm{GHz}$, as shown in Figure 4 .

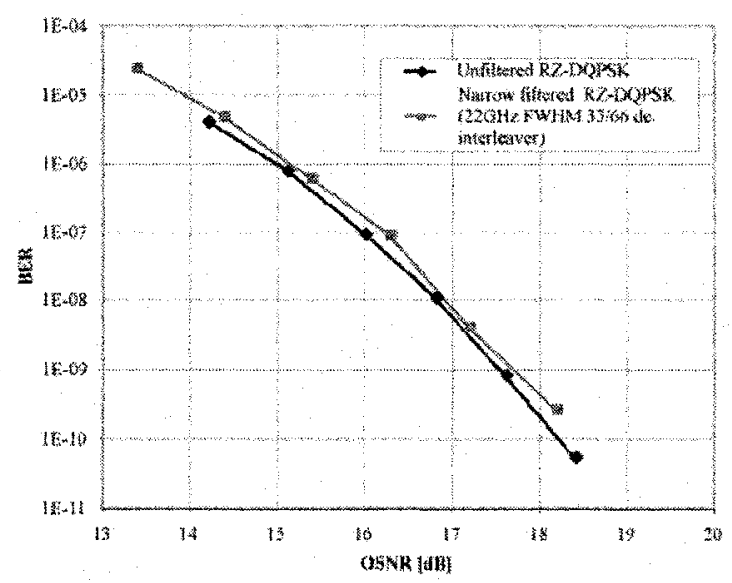

Figure 3. BER versus system OSNR for unfiltered (black diamonds) and narrow filtered (gray squares) RZ-DQPSK. Filtering is performed at the receiver by a $33 / 66 \mathrm{GHz}$ de-interleaver. No propagation.

As shown in Figure 5, when all the $6433-\mathrm{GHz}$-spaced DWDM channels are present, a penalty arises, depending on the relative state of polarization between the two closest channels. This penalty can be cancelled in case of orthogonal relative States Of Polarization (SOPs).

RZ-DQPSK signal performance over $2000 \mathrm{~km}$ in a $33-\mathrm{GHz}$ DWDM environment is shown in Figure 6. $1548 \mathrm{~nm}$ RZ-DQPSK channel is polarizationscrambled. Back-to-back data (blank square curve) are compared with BER after $2000-\mathrm{km}$ propagation (black square curve). As for previous measures, at the 
receiver channel is isolated from 64 DWDM copropagating channels by the cascade of the de-interleaver and optical band pass filter. Penalties due to propagation are evidenced: the phase modulation on the neighbor channels impairs propagation, owing to linear inter-channel cross talk due to PM spectral broadening and owing to non-linear interactions. As shown in figure, the experimented system, designed for standard IMDD propagation, is intrinsically limited in maximum achievable OSNR by the amplifier saturation output power. After $2000 \mathrm{~km}$ the maximum OSNR for the $64 \mathrm{DWDM}$ channels is $17.5 \mathrm{~dB}$.

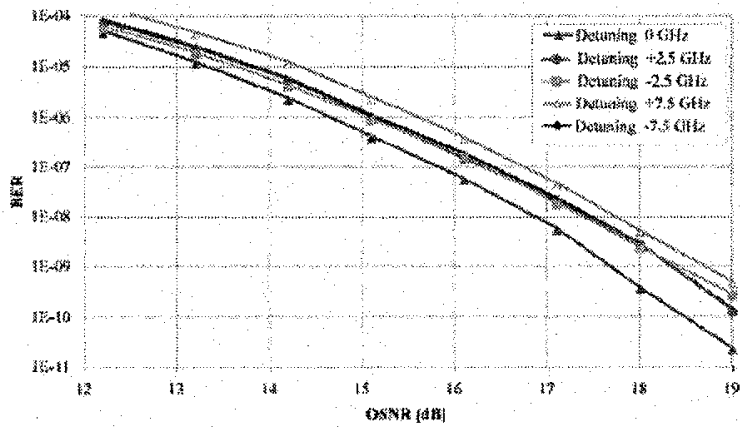

Figure 4. BER versus system OSNR for filtered RZ-DQPSK for signal wavelength detuning within $\pm 7.5 \mathrm{GHz}$ with respect to the de-interleaver optical transfer function. No propagation.

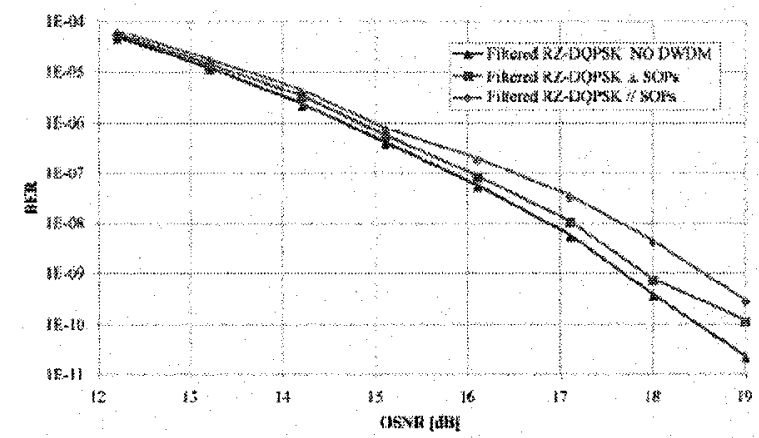

Figure 5. BER versus system OSNR for filtered RZ-DQPSK. When all $64 \mathrm{CH}$ are presents (gray squares and diamonds) penalty varies with relative SOPs of closest channels. No propagation.

Pre-filtering impact is evaluated as well by employing, as a tight pre-filter, an interleaver with the same spectral characteristics of the de-interleaver. Comparison between the two un-propagated curves (blank square curve without pre-filter and 
blank circle with pre-filtering) shows about $2 \mathrm{~dB}$ penalties. It is noteworthy that total filtering transfer function is non-optimized for DQPSK format. Gray circle curve represents $2000-\mathrm{km}$ propagation performance with pre-filtering. With respect to back-to-back curves, after $2000-\mathrm{km}$ propagation lower penalties (about $0.8 \mathrm{~dB}$ ) are found between pre-filtered (gray circles) and non-pre-filtered (black squares). Pre-filtering in fact limits the spectrum of DWDM channels broadened by phase modulation before propagation, thus reducing inter-channel cross talk.

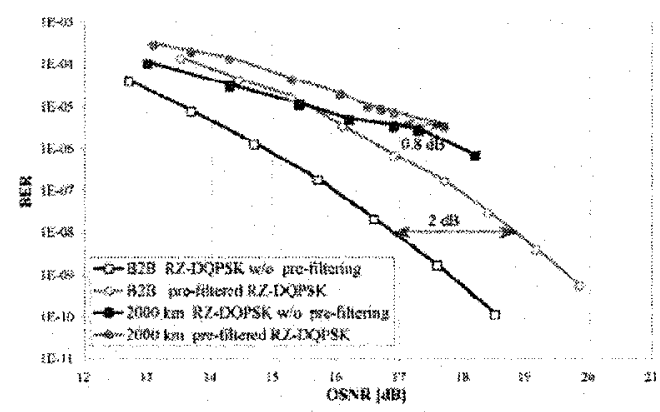

Figure 6. BER versus DQPSK channel OSNR in presence of the de-interleaver and of the 64 DWDM channels for back-to-back (blank squares) and after $2000 \mathrm{~km}$ (black squares); in presence of the de-interlaver, the 64 DWDM channels and the interleaver before propagation for back-to-back (blank circles) and after $2000 \mathrm{~km}$ (gray circles).

\section{CONCLUSIONS}

We have experimentally presented the impact of optical filtering on the transmission of a RZ-DQPSK signal at $20 \mathrm{Gbit} / \mathrm{s}$ equivalent bit rate in a 64-channel DWDM system. Propagation over $2000 \mathrm{~km}$ is obtained in a link not optimised for this type of modulation format. From these preliminary results, we expect that RZDQPSK degradation in ultra-long-haul transmission can be reduced by a suitable pre-filtering optimised for RZ-DQPSK modulation format in order to confine optical spectrum before propagation limiting non-linear interactions among channels. 


\section{ACKNOWLEDGMENTS}

The authors thank CoreCom Optical Technologies and Integrated Optics Labs for the project and the realization of the MZDI in collaboration with Pirelli Labs Milan, Italy, and Pirelli Submarine Telecom Systems - Milan, Italy for support during experiments.

\section{REFERENCES}

[1] T. Ono, Y. Iano, "Key technologies for Terabit/second WDM systems with high spectral efficiency of over $1 \mathrm{bit} / \mathrm{s} / \mathrm{Hz}$ ", IEEE J. Lightwave Technol., vol. 34, pp. 20802088, 1998.

[2] T. Hoshida, O. Vassilieva, K. Yamada, S. Choudhary, R. Piecqueur, H. Kuwahara, "Optimal $40 \mathrm{~Gb} / \mathrm{s}$ modulation formats for spectrally efficient long-haul DWDM systems", J. Lightwave Technol., vol. 20, pp. 1989-1995, 2002.

[3] C. Wree, J. Leibrich, J. Eick, W. Rosenkranz, "Experimental investigation of receiver sensitivity of RZ-DQPSK modulation format using balanced detection", in Proc. Optical Fiber Conference (OFC 2003), pp. 456-457, 2003.

[4] P.S. Cho, V.S. Grigoryan, Y.A. Godin, A. Salamon, Y. Achiam, "Transmission of 25Gb/s RZ-DQPSK signals with $25-\mathrm{GHz}$ channel spacing over $1000 \mathrm{~km}$ of SMF-28 fiber", IEEE Phot. Tech. Lett., vol.15, pp. 473-475, 2003.

[5] H. Kim, R.J. Essiambre, "Transmission of $8 \times 20 \mathrm{~Gb} / \mathrm{s}$ DQPSK signals over $310-\mathrm{km}$ SMF with $0.8-\mathrm{b} / \mathrm{s} / \mathrm{Hz}$ spectral efficiency", IEEE Phot. Tech. Lett., vol. 15, pp. 769-761, 2003.

[6] P. Boffi, L. Marazzi, L. Paradiso, P. Parolari, A. Righetti, D. Setti, R. Siano, R. Cigliutti, D. Mottarella, P. Franco, M. Martinelli " $20 \mathrm{~Gb} / \mathrm{s}$ differential quadrature phase-shift keying transmission over $2000 \mathrm{~km}$ in a 64-channel WDM system" Optics Communications to be pubblished

[7] P. J. Winzer, M. Pfennigbauer, M. M. Strasser, W. R. Leeb "Optimum Filter Bandwidths for Optically Preamplified NRZ Receivers" J. Lightwave Technol., vol.19, no.9, pp. 1263-1273, 2001.

[8] P. J. Winzer, S. Chandrasekhar, H. Kim "Impact of Filtering on RZ-DPSK Reception" IEEE Photon. Technol. Lett., vol.15, no.6, pp. 840-842, 2003.

[9] R.A. Griffin, A.C. Carter, "Optical differential quadrature phase-shift key (oDQPSK) for high capacity optical transmission", in Proceedings of Optical Fiber Conference 2002, WX6, 367-368, 2002.

[10] F. Morichetti, R.Costa, A.Cabas, M.Ferè, M.C.Ubaldi, A.Melloni, M.Martinelli "Integrated optical receiver for RZ-DQPSK transmission systems", Proceedings of Optical Fiber Conference 2004, FC8 (2004). 